\title{
ANÁLISIS DE LA FORMACIÓN DE LAS ENFERMERAS III. DE LOS MODELOS DE PROFESIÓN Y FORMACION A CONTRASTE
}

\author{
ANA M. Piera ORTS \\ Lic. Cc. de la Educación \\ Hospital General Universitario de Valencia \\ Correspondencia: Dr. Lluch, 159- $1^{\circ} .46011$ Valencia
}

\section{RESUMEN}

Se incluía en este estudio la descripción de los modelos de formación y profesional en enfermería, tomados en correspondencia a una realidad asistencial que se halla muy difundida en el caso profesional y en el de la formación los modelos descritos se ajustan al de la institución tomada como referencia, aunque no se les debe suponer únicos. Unas descipciones que permitirían, posteriormente, establecer los constrastes sobre la dirección de cada uno.

\section{EDUCATION OF REGISTERED NURSES: A DIAGNOSIS III. ABOUT EDUCATION AND PROFESSIONAL MODELS}

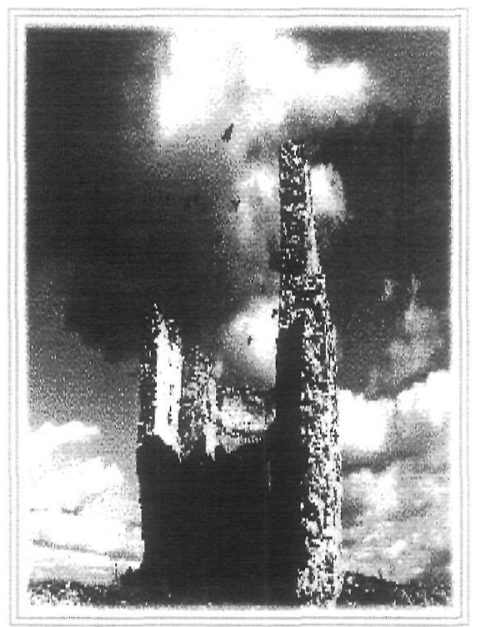

Paredón de San Miguel Carbonero el Mayor

\section{SUMMARY}

This paper compares the nursing education of registered nurses with the requirements of the nursing profession. Do they square up?

\section{INTRODUCCIÓN}

El modelo profesional debería, en principio, inspirar el tipo de necesidades formativas $\mathrm{y}$, a su vez, el modelo formativo debería tratar de posibilitar la mejora del ejercicio profesional. Algunas de las cuestiones, que tendrían que ver dentro de ese proceso de intercambio, estarían relacionadas con la identificación del origen del gobierno de la práctica profesional así como su regulación que, en caso de profesiones como la médica, se hace evidente que se regulan a través de los mecanismos de incorporación de nuevos conocimientos; en el caso de la enfermería este aspecto no se aprecia en su superficie.
Ambos modelos, se corresponden con una realidad asistencial que se halla muy difundida en el caso profesional y en el de la formación, el modelo descrito se ajusta al de la institución tomada como referencia, aunque no se les debe suponer único, tan sólo, diferenciados porque los diseños para la programación de actividades se han tomado, íntegramente, sin combinar con otros centros, aunque las bases reguladoras en que se apoya tienen una naturaleza generalizada dentro del territorio español.

Esta descripción nos permitirá, posteriormente, establecer los contrastes sobre la dirección de cada uno. 


\section{MODELO PROFESIONAL ENFERMERO.}

La representación del modelo profesional enfermero pretende ser fiel síntesis de la labor diaria de enfermería que, en general, puede encontrarse tanto en un hospital (instituciones cerradas) como en un centro de salud o centro de especialidades (instituciones abiertas). Una labor que, por operar dentro de un ámbito de relaciones humanas, con sujetos que son susceptibles de alguna pérdida de salud o padecen ya esa circunstancia, lleva implícita, además de sus particularidades que se derivan de los conocimientos que se aplican, una serie de consideraciones deontológicas que se mueven en el terreno de la bioética.

Para esta configuración del modelo profesional de enfermería, hemos contemplado una serie de regulaciones legales, distintos manuales de funcionamiento interno de hospitales, los protocolos de actuaciones enfermeras así como opiniones de profesionales del campo de la salud. Hemos considerado el contenido de una serie de trabajos, en los que se vierten opiniones, sobre la proyección hacia un futuro, que contemplen las nuevas formas que asumirá el papel de la enfermera.

La dificultad del modelo no consiste, tan solo, en elaborar una construcción que contemple todos los aspectos que puede abarcar el ejercicio profesional, sino también, en salvar el obstáculo que supone cierta falta de definición en determinados documentos legales, que son los pilares principales de todo modelo profesional. En el caso de enfermería en su reglamento vigente, en ocasiones, se prescriben tareas como si de funciones se tratara y de funciones como si de obligaciones se tratara igualmente. Ya conocemos que no todas las enfermeras ejercen como supervisoras, ni tampoco el conjunto de las enfermeras tienen las mismas funciones, dependiendo éstas, de la unidad a la que estén adscritas; pero el conjunto de enfermeras diplomadas, sí tienen asignadas, efectivamente, el mismo nivel de competencias en materia de salud, dentro de las instituciones sanitarias.

Cuando escribíamos sobre la institucionalización de la formación de profesionales sanitarios, vimos que ésta estaba estrechamente vinculada a regulaciones administrativas, derivadas de cambios sociales, tales como la mejora de las condiciones económicas o la nueva función hospitalaria.
Es lógico pensar que la representación de nuestro modelo profesional no se puede expresar partiendo, solamente, de las disposiciones legales en la actualidad sino que tenemos que considerar una serie aspectos que derivados de la Ley General de Sanidad de 1986, de los nuevos proyectos de reforma sobre las competencias de personal de los equipos de salud o incluso de las reivindicaciones de los profesionales de enfermería qu configuran ya, en cierto modo, un nuevo modelo profesional, que si bien no es el que realmente tiene lugar al completo en las instituciones sanitarias sí puede considerarse, en cierto modo, como el modelo acorde con la actualidad del servicio que tienen que prestar.

El modelo, tal y como aparece en la figura 1, recoge la función enfermera con esa dualidad que presenta a esa colaboradora con la función del médico, compaginada con su acción independiente de investigadora, docente y de responsable de cuidados.

Nuestro modelo parte del examen de cuáles son las funciones que, en general, se describen en la mayoría de los documentos enfermeros, bien de carácter legal o bien académico, así como de la consideración de su instalación en entornos organizativos. Hemos tenido en cuenta también, ese carácter cerrado que adquieren las profesiones en orden a ese pacto paradigmático por el cual en "recompensa por el acceso a su conocimiento especial, al profesional se le otorga un mandato especial para el control social campo profesional y un grado relativamente elevado de autonomía en la reglamentación de su práctica" (SCHÖN, 1992, pág. 41). Describiremos ahora el significado de este conjunto de funciones para acercarnos al tipo de exigencias que en el ámbito profesional representan. La función asistencial tiene una triple vertiente según se trate de unas tareas u otras. Dentro de la terapia de cuidados, puede considerarse cualquier actividad planificada por enfermería, encaminada a controlar las condiciones físicas y psíquicas de cada paciente y cuya valoración previa viene determinada por los conocimientos especiales adquiridos para su detección y solución. De las tareas derivadas de esta actividad, se seleccionan algunas de las rutinas y se delegan a personal auxiliar, si así lo valora la enfermera. La función de aplicaciones terapéuticas así como la de técnicas clínicas pueden derivar, en mu- 
chos casos, en actividades propias de la planificación enfermera, a partir de la valoración que se lleve a efecto sobre el paciente, pero no siempre será así; y podemos apuntar que, la mayoría de estas aplicaciones, serán a partir de prescripciones del médico responsable del paciente sujeto de estas ejecuciones. Considérese que las administraciones medicamentosas, aún cuando sean realizadas por enfermería, nunca son prescritas por otro profesional que no sea el médico responsable del diagnóstico y seguimiento del paciente. Lo que sí será responsabilidad de la enfermera, será la correcta aplicación terapéutica de todos los tratamientos farmacológicos, así como de todas las técnicas clínicas. Señalaremos que tanto las aplicaciones terapeúticas como las técnicas clínicas pueden ser susceptibles de realización a partir del seguimiento de un protocolo en el que se señala, de modo pormenorizado, el paso a paso, de cada una de las técnicas, no haciéndose explícito, en cada una de las ocasiones de su práctica, su efecto biofísico o biomecánico.

La función docente, extendida de forma general por todas las instituciones sanitarias, viene a ser la expresión práctica de acogida del alumnado de enfermería, así como de formación profesional, por el personal responsable de cada unidad, servicio o sección y que, con o sin plan de prácticas, los alumnos participan de la vida cotidiana de los profesionales con los que comparten tareas que unas veces son explicadas y fundamentadas $y$, otras realizadas con la inercia de cada día.

Finalmente, la función investigadora es en la actualidad una actividad que comenzó a extenderse con mayor amplitud, a partir de la entrada en vigor de la Ley General de Sanidad de 1986, por la que se concedía mayor autonomía a los colectivos de enfermería en lo que respecta a su papel relevante en los centros sanitarios, con las nuevas direcciones de enfermería. De todos modos, señalaremos que esta función, aún cuando se señala en los manuales de funciones de la mayoría de Centros y se promueven una considerable cantidad de certámenes y premios de investigación, no es una de las mayores actividades llevadas a cabo por los colectivos de enfermería, como se puede observar a través de las publicaciones períodicas de estos ámbitos profesionales. Estas tres funciones, aunque de igual relevancia dentro del modelo profesional, no tienen en la práctica real un ejercicio efectivo equilibrado, siendo la función asistencial la más extendida. Para una mejor descripción de este modelo, hemos tomado una serie de referencias de aquellos aspectos que nos han informado para la composición de su formato, a partir de documentos de trabajo, órdenes y normativas, que determinan la reglamentación de la práctica profesional del Hospital General Universitario de Valencia. Este centro sanitario, tiene una plantilla superior a 2.000 empleados, de los cuales 450 son enfermeras. Desde la observación y participación dentro de esta organización, se han tomado registros de las normas de funcionamiento, así como de las relativas a las prácticas profesionales de donde hemos recogido el modo en que son interpretadas por los mandos intermedios de las diferentes unidades de asistencia, así como del tipo de documentos que han de cumplimentarse día a día, como resultado de la función profesional y que a continuación describimos. De este modelo, obviamente, lo que se rescata desde la definición institucional es, precisamente, la función asistencial dado que, organizativamente, lo que aparece desde la perspectiva de trabajo remunerado es lo que hace referencia a la estructura del puesto de trabajo, esto es, el área asistencial. Un aspecto que recogemos a modo de paréntesis de relevancia ya que planea sobre todo el conjunto de elementos que afectan al tema. De los grupos que definen la práctica profesional, se derivan aspectos relativos a la socialización del profesional. La realidad organizativa del trabajo enfermero, dentro del territorio español, es que su función, que mantiene un cierto grado de independencia del médico, se ve abocada a moverse, prioritariamente, en el terreno asistencial de los actos médicos delegados en detrimento de su autonomía de cuidados, no tanto porque su papel no sea del todo necesario, tal y como se ha visto en el modelo profesional expuesto con el detalle de todas sus funciones, sino por la propia organización sanitaria, cuyo modelo asistencial más extendido es una combinación de la distribución de actividad por turno, dentro de la distribución de pacientes, agrupados por especialidades médicas, en la que, muy discretamente, se inicia la distribución de pacientes por enfermera. Y será dentro de esa modalidad organizativa, cuando la enfermera entre en contacto con los modos de "cuidar" que le sean encomendados 
en cada organización, siendo este momento cuando se inicie su socialización más efectiva, sustancialmente diferente del proceso que iniciará bajo la exclusiva supervisión de sus profesores, todos pertenecientes al Departamento de Enfermería.

\section{EL MODELO DE FORMACIÓN.}

Hemos tomado como referente principal la modalidad formativa del Hospital General Universitario. Este referente no es, en modo alguno, singular dados los relatos y afirmaciones que fuimos $\cdot r e c o-$ giendo a lo largo de nuestra indagación, que confirmaron la similitud entre la mayoría de los diseños formativos, con pequeñas variaciones en calendarios de prácticas y horarios de clases. En última instancia, señalaremos que las variantes están en función de la oferta de materias optativas o la disponibilidad de plazas en centros hospilatarios o ambulatorios. La modelización, que tomemos observada la realidad didáctica y la construcción teórica del proceso de formación, la perfilamos como referente esencial para realizar el contraste que buscamos. De los elementos que configuran el modelo profesional enfermero, hemos visto que además de la función investigadora y docente que precisa la puesta en práctica de procesos metacognitivos, en la función asistencial, la enfermera tendrá que enfrentarse a dos tipos de acción: a) aplicaciones terapeúticas sobre sujetos con pérdida de salud, combinadas con técnicas especializadas; b) valoración de atención clínica. En ambos tipos de acción, es necesario poseer unos conocimientos estructurados y conectados, de modo que permitan al profesional enlazar e identificar una serie de aspectos de la acción correcta que ha de acometer. Para este tipo de acciones se diseña un modelo de enseñanza mixta que, en su última etapa se basa en problemas, tomado de la E.U. Enfermería del Hospital General Universitario de Valencia, y aproximándolo al que proponen Barrows y Tamblyn (BARROWS y TAMBLYN, 1980; EDWARDS, BRANNAN, BURGUESS, PLANCHE y MARRIER, 1987), es la alternativa al aprendizaje memorístico, desconectado de la práctica de atención y cuidados enfermeros. Su valor se justifica considerando la forma en que se produce el razonamiento clínico, que parte de la información sobre el paciente, hasta llegar a las acciones orientadas a su mejora o curación (figura 2) Una expre- sión práctica didáctica recogida en nuestro Centro tomaba esta forma:

\section{a). Presentación de las materias teóricas}

Los contenidos de las diferentes materias se presentan de modo individual por cada profesor, adoptando formatos personalizados en éstos. Una dinámica parece haberse instalado en la Escuela, cuál si no existiesen otros formatos; son los mensajes unidireccionales apoyados en esquemas-resumen del temario seleccionado por los profesores y con gran soporte de transparencias.

b). Modalidad de trabajo en prácticas

1. Seminarios de prácticas. Se presentan en lugares no asistenciales, pero con materiales reales. Los alumnos observan al profesor que expone y luego realizan las tareas observadas.

2. Taller de casos prácticos. Cada alumno de tercero acude a dos seminarios, en los que se lleva a cabo una exposición de un caso práctico al grupo de alumnos. La duración es de dos horas. La presentación es unidireccional desde el profesor a los alumnos.

\section{Prácticas asistenciales.}

Los objetivos generales que se distribuyen a los supervisores o enfermeras responsables de las unidades, son los que se expresan en los Decretos reguladores de la formación de los Diplomados en Enfermería y que señalan que el sentido de esta actividad es el de crear experiencias clínicas, bajo el control del personal de enfermería y en los lugares adecuados para realizar cuidados de enfermería que haya de dispensar al enfermo. Además de estos, se expresan otros relativos a la adquisición de habilidades y destrezas en la realización de cuidados de enfermería o la posibilitación del desarrollo de habiliddes de carácter colaborador en los equipos. Se indican otras cuestiones como la de proporcionar conocimiento suficiente sobre la naturaleza ética de la profesión o proporcionar Educación Sanitaria al individuo y familia.

\section{Elaboraciones de los alumnos}

El alumno pone en marcha dos mecanismos de trabajo individual durante el curso académico. De un lado, en las clases teóricas cuyos contenidos quedaron recogidos en el apartado dedicado al Plan de formación, su actividad se ciñe a escuchar, copiar transparencias que se les muestras o tomar 
apuntes, estudiar de modo complementario en bibliografía o textos recomendados y a realizar ejercicios escritos de carácter nemotécnico, para superar las materias.

Una segunda vertiente del trabajo que se le solicita al alumno, es de naturaleza más personal. Cada alumno tiene su propio cuaderno de prácticas en el que registra aspectos como la estructura organizativa de la unidad o consulta donde realiza sus prácticas, el tipo de pacientes, Ios protocolos que va recogiendo y la descripción de su propia actividad. Para los alumnos de tercero, esta modalidad de trabajo lleva parejo la realización de un Plan de cuidados específico.

\section{EL CONTRASTE DE AMBOS MODELOS.}

En este apartado se aportan datos que proceden de una parte del cuestionario trabajado para el establecimiento y confirmación de la hipótesis de investigación. Una hipótesis de investigación.

Aparentemente parecen adecuarse en cierto modo, sin embargo, no se aprecia de modo explícito la modalidad real de las prácticas; por ejemplo, en los textos que recogen las entrevistas se cita reiteradamente una situación problemática con las prácticas de enfermería dentro de las Unidades asistenciales. Así mismo, la complejidad de funciones profesionales no parece concondar con los contenidos de los cuadernos de prácticas (material denominado como artefactos de investigación cualitativa), donde se recogían transcripciones literales de hojas de instrucciones y pequeños detalles, así como experiencias relativas a su papel como "la enfermera del doctor ....". Hemos constatado que no existe un modelo sobre la práctica enfermera que prevalezca sobre los demás. Esta ausencia se aprecia tanto directa como indirectamente en cualquiera de los apartados que hemos visto. El modelo profesional nos mostraba un marco de actuaciones de naturaleza institucional, que recogía una doble funcionalidad que se movía en la base de dos modelos de ejercicio profesional, de una labor informada e independiente y el vinculado a la figura del facultativo y dependiente de éste. Un ejercicio profesional que no gobierna su propia regulación y tampoco decide sobre la totalidad de sus funciones; doble funcionali- dad que pudimos apreciar en los datos que nos ofrecieron las encuestas. La organización de las clases teóricas y prácticas, cuyo diseño precisa de la colaboración directa de los profesionales de los centros sanitarios, no cuenta, realmente, con una estructura adecuada para el ejercicio docente, en la medida que los profesionales en ejercicio no están integrados en los programas de formación, exceptuando la figura del profesor asociado de prácticas.

La mirada clínica es "como un acto perceptivo subtendido por una lógica de las operaciones; es analítico porque reconstituye la génesis de la composición... La observación clínica implica dos dominios que le están asociados necesariamente y que se han conjugado entre sí: el dominio hospitalario y el dominio pedagógico" (FOUCAULT, 1991, p. 157). Pero, la oportunidad de vivir las situaciones reales de las personas que sufren cualquier patología, con los procesos de pérdida y recuperación o no de la salud que se produzcan, con la complejidad que supone percibir la configuración de ese proceso, no se le posibilita al estudiante que adquiere su formación, en gran medida, alejado de aquellas situaciones en las que pueda tener ocasiones reales de ejercitar la mirada clínica.

Otro aspecto de la planificación educativa, que se nos muestra muy directamente, es que ésta se reduce a un tipo de actividad docente de distribución de contenidos del área y de organización de tiempos como constatamos en el modelo de formación; pero en modo alguno pudimos percibir la existencia de un cierto nivel de formalización de las enseñanzas. En todo caso, sí percibimos la dificultad de "¿como enseñar la complejidad de situaciones que se dan en la práctica profesional?", como nos planteaba una de las profesoras que participó en nuestra charla compartida.

Lo que en suma, parece confirmarse es que la conexión entre ambos debería ser mucho más estrecha $y$, aunque se manifiesta una cierta tendencia a modificar el actual ejercicio profesional, especialmente, en el área de Salud Pública y, en cierto modo, recogiendo algunas de las escasas experiencias de autonomía profesional, como el caso de las enfermeras visitadoras del año 1931, como vimos en otro lugar. 


\section{BIBLIOGRAFIA}

BARROWS, H. S. and TAMBLYN, R. M. (1980) Problem-Based Learning. An Approach to Medical Education. Springer Publishing Company, Inc. New York. págs. 206.

EDWARDS, J.C.; BRANNAN, J.R.; BURGUESS, L.; PLANCHE', W.C. and MARRIER, R.L. (1987) Case Presentation Format and Clinical Reasoning: a Strategy for Teaching Medical Students. En: Medical Teacher, Vol. 9, No. 3, págs. 285-292.
FOUCAULT, M. (1991) El nacimiento de la clínica. Una arqueología de la mirada médica.Siglo Veintiuno de España Editores, S.A. $14^{\mathrm{a}}$ edición. Ed.. original 1963. págs. 293.

SCHÖN, D.A. (1992) La formación de profesionales reflexivos. Hacia un nuevo diseño de la enseñanza y el aprendizaje en las profesiones. Ed. Paidós. MEC. págs. 310.

Fig.l.-

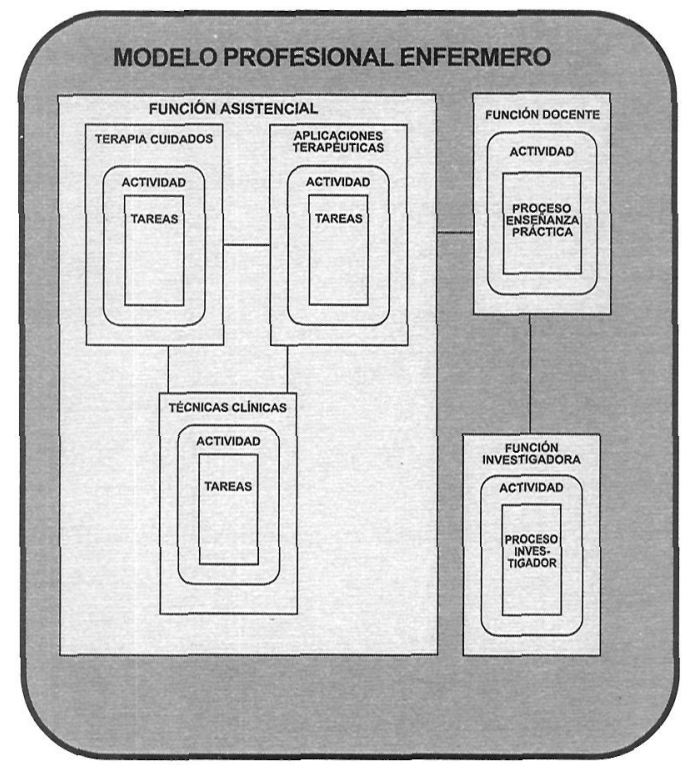

Fig.2.- Modelo de razonamiento clínico

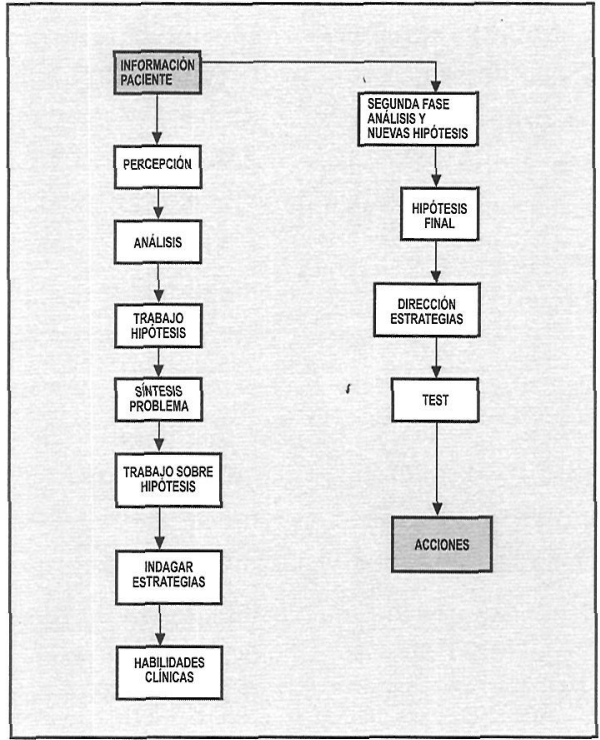

